This is a postprint version of the following published document:

Sánchez, M. Isabel, et al. Mobility management: deployment and adaptability aspects through mobile data traffic analysis. Computer communications, 95 (2017), pp. 3-14, December 2016

DOI: https://doi.org/10.1016/i.comcom.2016.04.012

(C) 2016 Elsevier B.V. All rights reserved.

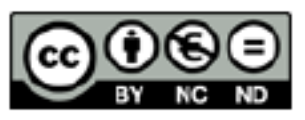

This work is licensed under a Creative Commons Attribution-NonCommercialNoDerivatives 4.0 International License. 


\title{
Mobility management: Deployment and adaptability aspects through mobile data traffic analysis
}

\author{
M. Isabel Sanchez ${ }^{\mathrm{d}, 1, *}$, Engin Zeydan ${ }^{c}$, Antonio de la Oliva ${ }^{\mathrm{b}}$, A. Serdar Tan ${ }^{\mathrm{c}}$, Utku Yabas ${ }^{\mathrm{s}}$, \\ Carlos J. Bernardos ${ }^{\mathrm{b}}$ \\ Simula Research Laboratory, Oslo, Nonway \\ ' Departamento de Ingeniería Telemática, Universidad Carlos III of Madrid, Madrid, Spain \\ 'Turk Telekom Labs, Istanbul, Turkey
}

Keywords:

Mobility

Handover

Distributed mobility management (DMM)

Big data

Traffic analysis

\begin{abstract}
A B S T R A C T
The expected boost in mobile data traffic and the evolution towards the next generation of networks are making cellular operators reconsider whether current approaches for handling mobility could be improved, according to the characteristics of the mobile traffic that actually flows through real networks. In this work, we make use of extensive analysis of real network traces to infer the main characteristics of mobile data traffic for a particular operator. Our analysis focuses on the features related to mobility, i.e., location information, number of handovers, or duration of the data traffic exchange. New techniques to gather the mobility characteristics of the user based on data and control packets correlation are designed and applied to compare the gains of deploying different mobility management approaches. We show that adapting the mobility management mechanism to the degree of mobility and the network characteristics brings some benefits to the network operator over the current approach, especially in scenarios of low mobility, where a dstributed mobility management solution proves to be more efficient.
\end{abstract}

\section{Introduction and motivation}

Cellular networks have evolved into extremely complex systems, where performance and behavior depend on the interaction of a multitude of logical modules. Coupled with this complexity, any analysis performed over these systems faces scalability challenges due to the size (in number of nodes) and amount of traffic served. The expected evolution of cellular networks forecasts an increase in both magnitudes, due to the growing traffic demands and the so-called radio access network (RAN) densification. In this scenario, operators struggle to monitor and analyze their networks through an amalgam of vendor specific probes and management systems, providing information which is difficult to aggregate and analyze. The lack of tools for the design and optimization of next generation networks, carrying several orders of magnitude more traffic and serving a wider set of possible clients (including machine and humans) is a challenge, requiring novel techniques that are able to provide trends, relations and design guidelines for the

\footnotetext{
- Corresponding author.

E-mail addresses: isabel@simula.no (M.I. Sanchez). engin.zeydan@turktelekom. com.tr (E. Zeydan), aoliva@it.uc3m.es (A. de la Oliva), tans@mef.edu.tr (A.S. Tan) utku.yabas@partner.turktelekom.com.tr (U. Yabas), cjbc@it.uc3m.es (C.J. Bernardos).

1 This work was carried out while M. Isabel Sanchez was at IMDEA Networks Institute and University Carlos [I].
}

deployment of new systems. A promising trend in this area is the use of Big Data techniques to gather information on the behavior of the network, analyzing and inferring knowledge out of the myriad of data flows transported by the network [1]. It is a common practice to monitor the traffic flowing through the network to evaluate network performance or to look for delay or usage patterns [2]. With the increase of data traffic and the raise of more powerful processing techniques and capabilities, this traffic monitoring has turned to Big Data techniques to analyze the traffic [3]. In this work, we built on top of these tools to provide some insights on the usage of resources for mobility management with actual data from a real operator's network.

The work reported in this paper started as a discussion on how to analyze the benefits and drawbacks of new techniques for traffic offloading in a real operator environment, specifically if the application of mobility protocols based on the Distributed Mobility Management (DMM) concept was worth the effort considering the structure of the network, deployment characteristics and the real user traffic. In order to answer this question, we tried to directly analyze the data traffic in one of the operator's core interfaces looking for mobility patterns and traffic characteristics, required to understand the performance of the mobility protocol. Through this direct approach we found two main problems: i) The overwhelming number of flows going through the interface and their lack of 
information matching location of the user and ii) the need for a mechanism to correlate the information carried in the data path and the control path, to match flows to users and cells. To overcome these challenges, we resorted to the deployment and analysis of the data using Big Data analytic techniques. Therefore, this paper focuses i) on the development of a platform to perform Big Data analysis over packet traces in a real operator environment in Turkey under privacy and regulation concerns, and ii) to showcase the utility of this new framework by the analysis of the suitability of applying a new mobility management concept, DMM, to the current network deployment. In order to gain an understanding of the whole network operation, this work also reports on the challenges of working with packet captures taken on the data path of the operator's core, which can carry thousands of flows, and the need of correlating these flows with the control data obtained from a different interface in the operator's core.

Following these ideas, the remaining of the paper is structured as follows: Section 2 presents recent works in the area of distributed mobility management and traffic analysis that are relevant for this work. Section 3 describes the method for the collection of the mobile data traffic information, which is analyzed in Section 3.4. Based on the results from these experimental measurements, in Section 4 we apply a DMM mechanism and compare it to the current deployment showcasing that it can be more convenient under certain mobility scenarios. Finally, Section 5 presents the final conclusions.

\section{Related work}

Managing the mobile traffic is becoming increasingly complex, both technically and economically for mobile operators. This is not only because of increased data usage and emergence of new services and models, but also due to the change in user's behaviors towards increased mobility. Mobility management support in cellular networks has always been a critical capability. Therefore, there has been numerous works outlining different mobility management approaches in the literature [4-9]. A recent comprehensive tutorial on mobility management in data networks that especially focuses on network mobility and session migration is given in [4].

Current architectures for mobile and cellular networks are centralized [6,7] and hierarchical [8] which drives the user traffic to go all the way up into the core network. Proxy Mobile IPv6 (PMIPv6) [7] and GPRS Tunneling Protocol (GTP) [9] protocols have been adapted for the applicability of the centralized mobility management solutions for mobile operators. Even though centralized mobility solutions reduce the signaling between the hosts and are relatively simple for following user's movements, there are various issues with their adoption in cellular networks as identified in [10]. Some of these problems are: low scalability due to the need for new mobility anchors as the number of mobile nodes and data traffic increase, per node mobility support that increases congestion on mobility anchors due to mobility support for all flows, single point of failure due to many mobile nodes connection to single mobility anchors [11], and non-optimal routes which may result in longer delays and excessive load in the core network.

As opposed to centralized solutions, distributed mobility management approach has proposed a flat and flexible architecture by ensuring per flow mobility support and optimal path routing in a highly scalable manner $[4,10,12,13]$. A comprehensive overview of distributed mobility management techniques that includes standardization activities of both the 3rd Generation Partnership Project (3GPP) and Internet Engineering Task Force (IETF) has been given in [4]. The standardization activities for distributed mobility management are primarily driven by IETFs DMM work- ing group [12]. The requirements for distributed mobility management have been recently given in RFC 7333 [10]. Some of these requirements constitute functionalities such as distributed processing, transparency to upper layers, IPv6 target deployments, re-usage of existing mobility protocols, co-existence with existing network deployments and hosts, by-passable mobility support for each applications and security considerations. From a practical implementation aspect, the authors in [13] have demonstrated the first practical evaluation results of distributed mobility management approach based on evaluations with real Linux-based prototype implementations.

Previous work leverage mobile traffic data towards mobility analysis based on various categories including analysis based on movement of individuals [14-16], understanding the resource consumptions $[15,17,18]$ for designing technological solutions and validation of the results obtained through mobile traffic analysis $[19,20]$. The studies in [14] and [15] demonstrate that the mobility of users in terms of the number of cells they visit can be low and the distribution turns out to be heavy-tailed, which shows the existence of certain users that visit hundreds of cells. The authors in [16] have investigated ways to identify large-scale social events by using the large variations in mobile traffic volumes. For validating purposes, such as population distributions over geographical areas, approximation of actual subscriber trajectories or real-time estimation of population levels, the authors in [14,19] have relied on the usage of mobile traffic data. Characterization of individual [18] as well as aggregate [15] access network traffic in cellular networks have been studied separately. Through traffic analysis, the authors in [18] identify flows related to mobile apps automatically by continually learning the apps distinguishing features. Finally, for more details of the recent studies on mobile traffic analysis, we refer to [21] where the authors have provided a thorough survey and categorization of mobile traffic analyses of different operator's data which are mostly collected from various parts of the operator's network infrastructure.

Big data and data analytics are recently emerging to facilitate the development of new analytics applications, and to leverage the mobile operators understanding and exploitation of data which is constantly flowing through their network infrastructures. In a more general point of view, one can find big data platform utilization (namely Hadoop [22]) and exploitation of data analytics by telecom operators in different recent studies [23-27]. For example, application areas of big data analysis applied by telecom operators ranges from anomaly detection for IT infrastructure security and resiliency [24], network coverage analysis [23], proactive caching for $5 \mathrm{G}[25,26]$ to social network analysis for consumer behavior modeling [27]. Authors in [28] propose a platform for mobile data network analysis that also monitors the traffic in the Gn interface and, in the case of LTE, in S-11 and S-1U interfaces using both Hadoop and Spark for storage and post-processing of the data collected by the probes. They focus on the information in GTP-C messages, describe their platform and provide a very light evaluation on the information that can be extracted from their probing, which could be further exploited. However they state the possibilities that this kind of approach open for mobility and performance assessment in an operators network, which is the key feature that we aim at evaluating by monitoring GTP control and data information.

However, none of the studies described above have demonstrated the deployment and adaptability aspects of DMM-based solutions inside a real mobile operator by exploiting a comprehensive mobile data analysis. Therefore, it is clear that in order to embrace new technologies for long term alternatives to current centralized cellular infrastructures, recently proposed DMM solutions need to be further investigated in the context of applicability 
and adaptability for mobile operators. Based on this observation, in this article, we focus on the validation of DMM approach's performance results using real operator's network data by exploiting big data techniques for data analysis. The data management (extraction, analysis and usage) process studied in this paper is specific to our distributed mobility management scenario and plays an important role in supporting the applicability of the proposed solution. As far as we know, the comparisons of distributed mobility management with current centralized mobility management approaches with large amount of mobile data usage are not available in the literature. Using the analysis in this paper, interesting conclusions are deduced by bringing real world considerations about the applicability of the new distributed mobility management solution inside a mobile operator's network.

\section{Description of the system}

As explained in Section 1, this work aims at providing an analysis of the suitability of applying a new mobility management concept, DMM, to the current network deployment. In order to do so, we needed to develop a new system to be able to i) gather user data traces, ii) correlate the overwhelming set of user and control information to extract relevant mobility information and iii) use the mobility information extracted to apply an analytical model for the packet delivery cost of DMM and compare it with current deployments. In the following sections we explain each of the steps in this complex process.

\subsection{Dataset and collection of information}

Identifying the structural patterns in the data traffic is of high importance for mobile operators in order to apply optimal mobility management techniques within their network. Mobile network traffic has a highly complex and massive structure, making it tough to analyze and reveal structural patterns. It is not unusual that one or more terabytes of data per second is flowing in a typical mobile operator consisting of 10 to 20 million subscribers which translates into roughly exabytes every month. The scale of this problem rules out direct sniffing approaches [29], with the additional problem of data packets not carrying information regarding the location of the user. The analysis of mobility requirements necessitates the extraction of handover related information, which can be tracked both in radio access and core network nodes. Accessing handover related information from the radio access nodes is difficult since the amount of probes that needs to be placed into the infrastructure can be large. Moreover, log data from different entities, such as the radio network controller (RNC), can be too hard to extract for further mobility analysis due to the unavailability of appropriate tools, which are mostly vendor specific. In High Speed Packet Access (HSPA), the core network is only notified of Location Area (LA) and Routing Area (RA) updates when the mobile terminal is in the idle state, since in connected state the access network can still locate it and report to the core network when necessary. Moreover, our approach aims to link user roaming across the network with the characteristics of the mobile traffic being exchanged.

Due to the aforementioned problems, we propose a system based on the extraction of handover related information observed in the core network nodes, where the correlation of the control message headers with the information of the flows in the user data plane is obtained by capturing the data and control packets in the $\mathrm{Gn}$ interface. One of the constraints imposed in the system design was the lack of existing measurement tools over the interfaces for mapping flow information with the location information inside the operator domain. The current infrastructure allows capturing control packets in the 3GPP $\mathrm{Gn}$ interface between the
Gateway GPRS Support Node (GGSN) and the Serving GPRS Support Node (SGSN) (see Fig. 1). Specifically, we track the Create Context and Update Context messages of the Packet Data Protocol (PDP) [9]. In this way, we can simplify the requirements for monitoring user mobility avoiding the need for multiple probes. For our analysis, we do not need to track the exact location of the user at the precise moment that it happens, but detect the changes in their point of attachment and characterize the traffic being exchanged by roaming users. We focus our analysis on handovers involving RA changes, which is inline with the current DMM architectures discussed in the IETF. ${ }^{2}$

The final outcome of this process provides a combined and detailed listing of the control and user plane packets indicating location of mobile terminals via the information included in PDP Create Context and Update Context messages. This trace is further processed to characterize mobile data traffic (see Section 3.4) and to obtain insights on the applicability of the DMM concept in an operator's network, as explained in Section 4.

\subsection{Architecture of the dataset collection mechanism}

A general view of the architecture for the extraction of flow information is provided in Fig. 1. The system is composed of mainly two elements, the Flow Extraction Manager (FEM) and the Processing Cluster that has been implemented using Hadoop [22]. One of the existing $\mathrm{Gn}$ interfaces with high traffic in the core network between SGSN and GGSN is mirrored and collected in the FEM, which applies initial processing and transfers the data to be analyzed and filtered into the Hadoop Cluster. The extracted flow information is sent back to FEM from the Hadoop Cluster for collecting analysis results. The analysis results are outputted by FEM for detailed observations of the network state. The details of the flow extraction process as well as the analysis in Hadoop cluster are given in Section 3.3. In the following, we detail some of the operations of the system.

\subsubsection{GTP-U and GTP-C correlation on Gn interface}

Network packets sent from a user equipment (UE) to the packet data network (PDN), e.g. Internet, pass through the SGSN which tunnels them towards the GGSN. GTP is used for tunneling the packets in the $\mathrm{Gn}$ interface [9].

The operation of GTP protocol differentiates user and control planes. The user plane packets on the Gn interface flow on the GTP User (GTP-U) [9], which is in effect a relatively simple IP based tunneling protocol allowing several tunnels between each set of end points. When used in an HSPA network, each subscriber will have one or more GTP tunnels, corresponding to each active PDP context, as well as possibly having separate tunnels for specific connections with different quality-of-service (QoS) requirements. Each tunnel is identified by a tunnel endpoint identifier (TEID) in the GTP-U messages, which should be a dynamically allocated random number. The control plane packets on the $\mathrm{Gn}$ interface are encapsulated on the control section of the GTP, namely GTP-C [9]. When a subscriber requests a PDP context, the SGSN will send a Create PDP Context Request GTP-C message to the GGSN giving details of the subscriber's request. The GGSN will then respond with a Create PDP Context Response GTP-C message which will either give details of the activated PDP context or will indicate a failure and give a reason for that failure. We make use of a Hadoop based platform (explained below) to aggregate packets in flows and correlate GTP-U and GTP-C information (described in Section 3.3). In this way we infer the mobility characteristics of the flows and associated GTP tunnels.

\footnotetext{
2 Note that routing areas may include one to several groups of cells connected to the same RNC.
} 


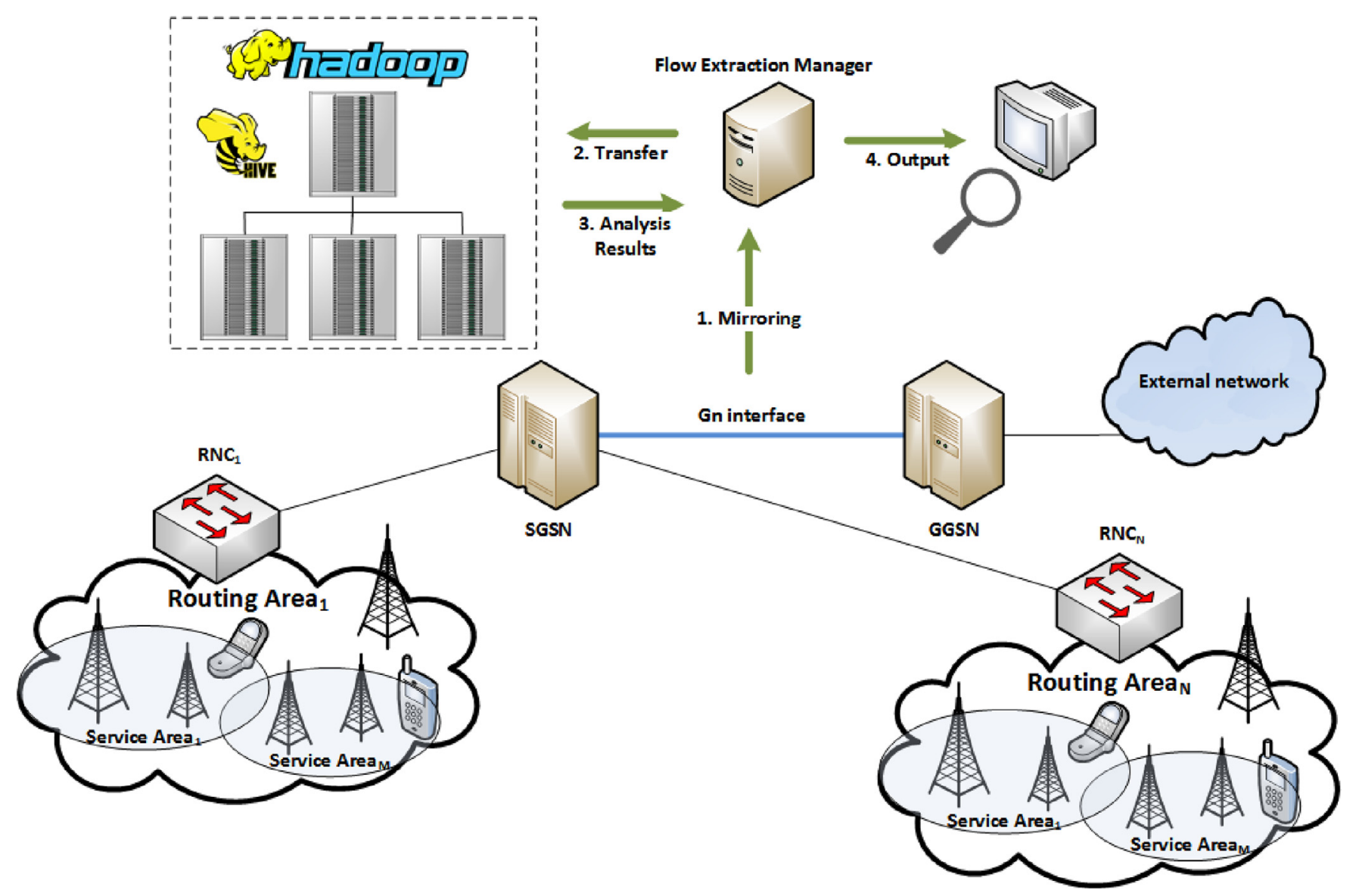

Fig. 1. Flow information extraction architecture.

\subsubsection{Mirroring of Gn interface}

The network of interest in this paper consists of an area covered by 10 SGSNs. The average total traffic over all regional areas consists of approximately 15 billion packets in the uplink direction and 20 billion packets in the downlink direction daily. This corresponds to approximately $80 \mathrm{~TB}$ of data flowing in uplink and downlink daily in the mobile operator's core network. The importance of this work can be seen by the exponential increase in data traffic that has to be handled by a mobile operator. For example, in 2012, the approximate total data traffic was over 7TB in both uplink and downlink daily. The packets are captured by a mirroring device which was already in place on the operator premises as part of their already deployed measurement system. Hence, this work takes benefit of already deployed probes without requiring any additional deployment. We tested our method on real-world Gn interface Internet traffic data where the flow traces obtained from the mobile operator are collected by a server on a high speed link of $200 \mathrm{Mbit} / \mathrm{s}$ for any requested time interval.

\subsubsection{Hadoop platform}

Among the available Big Data platforms, Hadoop [22] stands out as the most notable one as it is an open source solution. It is made up of a storage module, namely HDFS and a computation module, namely MapReduce. Whereas HDFS can have centralized or distributed implementations, MapReduce inherently has a distributed structure that enables it to execute jobs in parallel on multiple nodes.

The Hadoop cluster in our platform was implemented based on Cloudera's Distribution Including Apache Hadoop (CDH4) [30] version on four nodes with Intel Xeon E5-2670 CPUs, 32 cores, 20 TB hard drive and 132 GB RAM.

\subsection{Flow information and mobility characteristics extraction}

The process proposed in this work, extracts and matches the user data TEID (TEID_DATA) field in the GTP-C messages (Create and Update PDP Context) with the TEID in GTP-U packets, in order to add the corresponding location information to the traffic in the user plane. ${ }^{3}$ GTP-C messages include an Information Element containing the location of the mobile terminal, expressed by the Cell Global Identication (CGI), which is formed of the Mobile Country Code (MCC), the Mobile Network Code (MNC), the location area code (LAC), (which corresponds to the Routing Area identifier) and the cell identification ID (CELL-ID) or service area code (SAC) in Universal Mobile Telecommunications Service (UMTS). The service area (SA) identifies an area of one or more cells of the same location area and it is identified with a SAC, unique within that location area. As the MCC and MNC will remain unchanged for every packet in our trace, we monitor the LAC and SAC fields. A LA is a set of cells, which are grouped to decrease signaling overhead. Larger LAs reduce signaling for location updates because users hardly move out of the LA. However, the overhead introduced by paging is very high because there are many cells. This may be acceptable for circuit switched services, such as voice calls but it introduces a high delay on packet based communications. Therefore, a new definition, the Routing Area is introduced in packet switched services. The RA has the same conceptual functionality as the LA but its size is usually smaller. The differentiation between the RAs and LAs depends on the decision of the network operator. Typically, tens or even hundreds of base stations are present in a given

\footnotetext{
${ }^{3}$ Note that this location information is not carried in the data plane packets.
} 
LA. As we work with data transmissions, we will use the term RA from now on to refer to the greater area that groups several SAs.

A TEID uniquely identifies a tunnel endpoint on the receiving end of the GTP tunnel [9]. A local TEID value is assigned at the receiving end of a GTP tunnel in order to send messages through the tunnel. The GTP-C packets contain the information that identifies the location of the user (LAC and SAC fields) as well as a TEID_DATA field, which points to the identifier of the corresponding tunnel in the data plane. We extract from the GTP-U packets, which are identified by their TEID, several information regarding the characteristics of the mobile traffic, such as packet size or duration of the flows. The flow information can be extracted from data packets by taking into account simply five tuples namely, layer 4 protocol information (e.g. transport control protocol (TCP), User Datagram Protocol (UDP), Internet Control Message Protocol (ICMP), etc.), source and destination IP address and source and destination port numbers. Taking advantage of their uniqueness and matching the values of TEID and TEID_DATA fields on both data and control planes respectively, the GTP-C and GTP-U information is joined by the Hadoop cluster through successive map and reduce operations, to obtain a merged table showing the flow and CELL-ID of the user flows. The complexity of the operation comes from the fact that, in a given PDP session with a specific TEID, there can be multiple flows (although each TEID belongs to a specific user). This must be taken into account while processing the data.

The result of the processing of the data mirrored from the Gn interface is a collection of anonymised rows, each representing a flow, providing information regarding the time it is collected, its size, a flow identifier, TEID and unique $<$ SAC-LAC $>$ information. This last tuple uniquely identifies the location of the user generating the flow. This information is obtained by filtering packet information and aggregating it into flows, later this aggregated information is processed through several map reduce operations to merge the information coming from the control and data plane. The mechanism is applicable to all mobile operators using the 3GPP standard Gn interface and can be put into practice immediately, since it does not require the change of the monitoring platform of the operator.

\subsection{Mobile data traffic analysis}

In this section, we analyse the main characteristics of our dataset, built from the information captured at the Gn interface, between SGSN and GGSN. Network packets sent from a user terminal to the PDN, such as Internet, go through SGSN and GGSN where GTP constitutes the main protocol in network packets flowing through $\mathrm{Gn}$ interface, as described in Section 3.1.

We have collected two $1 \mathrm{~h}$ traces at times with different traffic load in the network. The "peak" and "low" hours are chosen among the $1 \mathrm{~h}$ intervals of a day that yields highest and lowest number of flows in our data set, respectively. In this study, the "peak" and "low" hours represent the intervals 8-9 pm and 4-5 am in a day, respectively. The peak and low hours are calculated via an on-line flow extraction manager tool which counts the number of flows passing through the Gn interface that is deployed inside the mobile operator's core network. Note that we have chosen the number of flows as metric for the peak and low hours since our analysis is focused on the user mobility and not on the overall amount of traffic generated in the network.

We utilized only two $1 \mathrm{~h}$ traces of data to analyze the mobile traffic due to the existence of high time correlation for network load. The study in [15] shows that there exists a high degree of temporal correlation where the high peaks occur at $24 \mathrm{~h}$ intervals and low peaks occur at $12 \mathrm{~h}$ intervals based on the calculated
Table 1

Summary results from the data traces.

\begin{tabular}{lll}
\hline & Low hour & Peak hour \\
\hline Total duration of the traffic trace & $1 \mathrm{~h}$ & $1 \mathrm{~h}$ \\
Total number of control packets & 2722234 & 4294077 \\
Total number of data packets & 18978264 & 28582691 \\
Total number of flows & 1655550 & 2982064 \\
Flows analyzed & 1054566 & 1926062 \\
Total number of control TEIDs in C-trace & 762102 & 1259128 \\
Total number of data TEIDs in C-trace & 671078 & 1020178 \\
Total number of TEIDs in U-trace & 83510 & 154650 \\
Total number of data TEIDs analyzed & 60932 & 116529 \\
Number of data TEIDs that experience handover & 1464 & 2413 \\
Total number of flow handovers & 7402 & 18854 \\
Total number of TEID handovers & 2624 & 4750 \\
TEIDs without handover in data trace & 59468 & 114116 \\
& $(97.6 \%)$ & $(97.9 \%)$ \\
Data flows without handover in data trace & 1047380 & 1907759 \\
& $(99.3 \%)$ & $(99 \%)$ \\
\hline
\end{tabular}

auto correlation function. This result is consistent with the diurnal (non-stationary) human activity patterns. Although there exists a high degree of correlation of network load at the same time of the day, these good correlation results (neither positive nor negative) are not reflected on the individual base station loads where the periodicity is missing. This shows that aggregate network load information, as we use in our study herein, has more predictable behaviour than local base station traffic characteristics. The results presented in [17] also indicate the strong dependence of traffic volume on previous states that lag by multiples of $24 \mathrm{~h}$ which is similarly due to the diurnal nature of the cellular operator's core network data. Similar results of periodicity have also been identified in [31] where Internet Protocol (IP) traffic volume in 7-day traces (including weekends) shows stable distributions and high correlation on lags of $24 \mathrm{~h}$ hence allowing further analysis on smaller scale traces. The results in these previous studies clearly show that appropriate sampling of the mobile traffic data can approximately yield sufficient statistics for our analysis.

Table 1 gathers the main characteristics of our dataset, after parsing, filtering and properly grouping the information available in the trace file. First of all, we present the total amount of data and control information that we have analyzed, expressing it in terms of flows and tunnels, which will be the main unit used in our mobility analysis. In light of our measurements, with more than a million flows ${ }^{4}$ spread over more than 80 thousand data tunnels, approximately $99 \%$ of flows and $98 \%$ of tunnels do not experience a handover. Note that the operator experiences overhead due to mobility management for these tunnels (mobility is provided by default in current operator networks), although no mobility is required by them. It also calls our attention the number of control messages exchanged linked to data tunnels (identified by the TEID_DATA field) that are not exchanging any data packet. That translates into a considerable overhead in the already challenged operator's network to maintain context for data tunnels that are not being used for any data transmission. Data flows, although more numerous than tunnels, have shorter duration, which results in a lower average number of handovers per flow than per tunnel. Note that the total number of TEID handovers in Table 1 aggregates the number of handovers from all the tunnels analyzed.

Fig. 2 shows the number of handovers per tunnel for the low hour (Fig. 2a), and peak hour ( Fig. 2b) performed in our dataset. We have included for comparison the number of tunnels and flows

\footnotetext{
${ }^{4}$ Note that the "number of flows analyzed" in Table 1 refers to the number of flows that include useful information for our latter mobility analysis, not the total of flows in the network.
} 


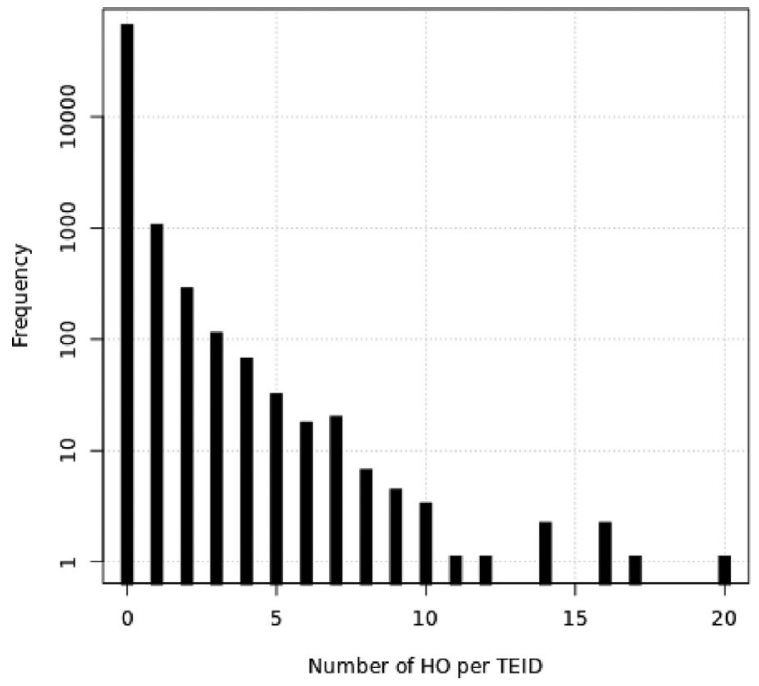

(a) Low hour trace

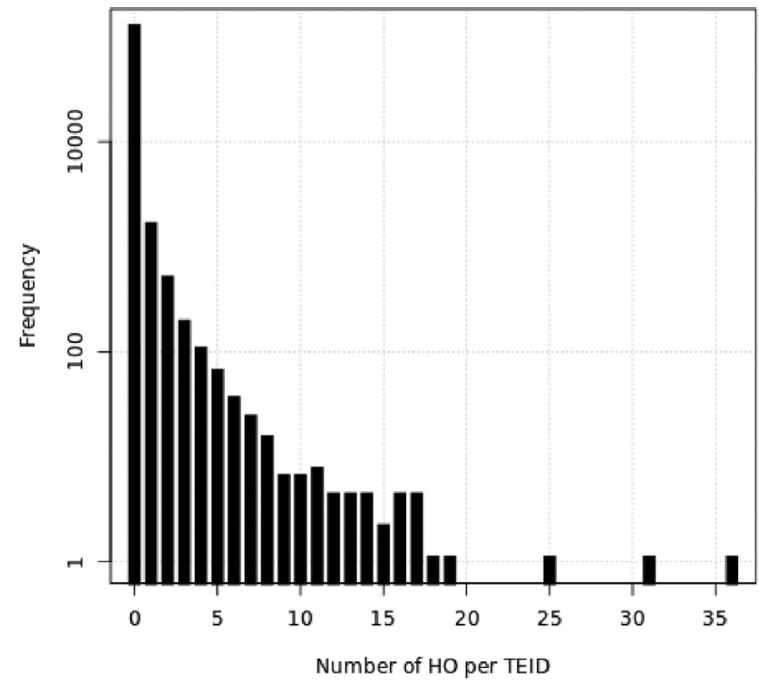

(b) Peak hour trace

Fig. 2. Histogram for the number of handovers performed per tunnel for the low and peak hours.

not moving in the network (tallest bars in $\mathrm{x}=0$; note the histogram in logarithmic scale). In the peak hour, the number of handovers increases, even though the higher number of RA changes are not frequent.

With regard to mobility, we highlight the high percentage of data traffic in the network that does not experience a handover (99\% of flows and almost 98\% of tunnels). Still, this traffic is provided with mobility support, just as any other in the network, which leads to an inefficient usage of resources in current network deployments, where mobility is granted to all the traffic being transmitted. Therefore, future network deployments could take into account these mobile data characteristics to improve scalability and free network resources.

We do not evaluate the changes of SA, first of all, for similarity to an initial deployment of DMM, which would be done by replacing packet gateway (P-GW) or serving gateway (S-GW) [32] (terms for GGSN and SGSN in LTE nomenclature). In addition, SAs are groups of cells, and they can overlap, which could lead us to misleading conclusions about user mobility. In any case, and just to confirm that the low mobility between routing areas is not due to the size of the area considered, we have measured the SA changes within a given RA. The number of tunnels that changes SA accounts to $5.6 \%$ of the tunnels analyzed in our dataset, which confirms our previous conclusion and the low mobility in the traffic analyzed.

Fig. 3 represents the duration of tunnel identifiers and the median of the time between routing area handovers for every tunnel. As shown in Fig. 3 the points are concentrated in the lower X and $Y$ range, meaning that from the flows experiencing a handover, there are a majority which are short lived and highly mobile. It can also be seen how the points form several lines across the plot (e.g., $X=Y$ ). These lines correspond to the points which ratio between the average time between handovers and TEID active time is constant. In fact these lines show the number of handovers that are more common among the users. The first of the lines (starting from the top of the graph) corresponds to tunnels surviving one handover. It is also worth explaining the reason behind the lack of data for $X<Y$. We measure the time between handovers through the changes in LAC and SAC of the active tunnels, hence for $X<Y$ the tunnel is already disconnected and data is not available. To finalize the comparison of the different traffic patterns for the peak and low area, Fig. 4 shows the CDF of the Residence Time (time between RA handovers per TEID) and Prefix Lifetime (the duration of a given TEID) for both hours. Fig. 4 only includes the data for flows experiencing a handover, which is a very reduced number of flows in both traces. As shown in Fig. 4 both traces exhibit very similar patterns. The peak hour shows a slightly higher residence time while the prefix lifetime is practically the same.

\subsection{Discussion on usage of LTE networks}

Note that a similar analysis can be performed with Long Term Evolution (LTE) as well. In LTE, an EPS Bearer context in the S5/S8 interface (S-GW - P-GW) includes control and user plane TEIDs (for GTP-based S5/S8) or GRE keys (for PMIP-based S5/S8) [33]. In both architectures, traffic is routed through a centralized network element that acts as the mobility anchor. However, GTP seems to be the dominant solution of the S5/S8 for mobile operators due to following reasons [34]:

- GTP based mobility solution is the most cost-effective and the least complex to integrate legacy and existing 3GPP networks which occupy about $90 \%$ of mobile subscriptions market share worldwide.

- GTP is a natural extension of the existing infrastructure (required on the S1 interface as well) and gives less work to stick with for mobile operators.

Moreover, as stated in [35], many operators have co-located GGSN and P-GW, which allows information gathering on $3 G$ and LTE at the same time making it easy for extension of the paper's results into LTE domain. Hence, the studies performed in this paper can be directly applied by mobile operators that deploy GTP based S5/S8 interface.

\section{Understanding DMM deployment on current networks}

In this section, we briefly explain how PMIPv6 and networkbased DMM work and evaluate the usage of DMM as an alternative to current deployments and we use the mobile data traffic characteristics already presented to back the reasons behind our comparison with an example from actual data. As we have explained in Section 3.1, the tunneling protocol chosen to handle mobility in the UMTS network is GTP. However, given the increasing bandwidth demand, the characteristics of the mobile data traffic and 


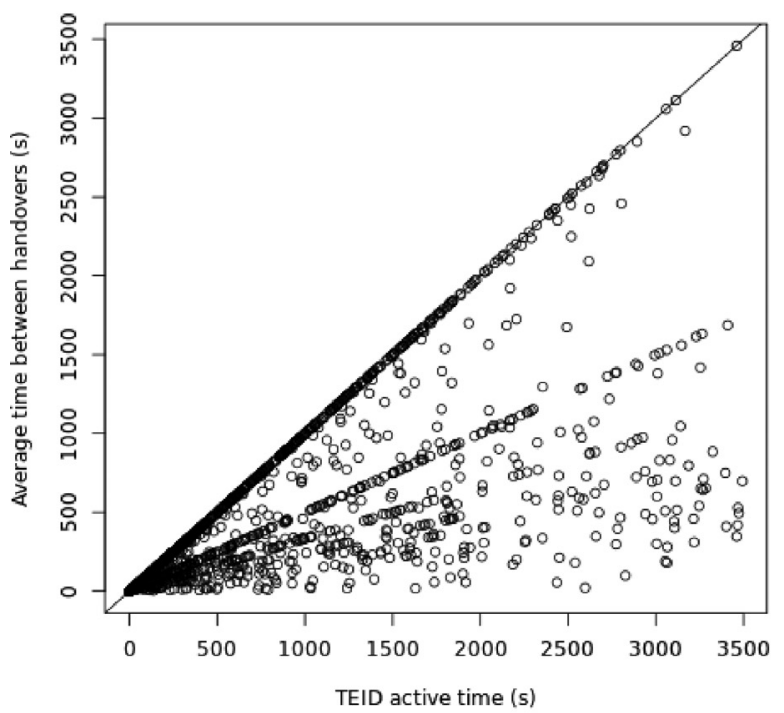

(a) Low hour

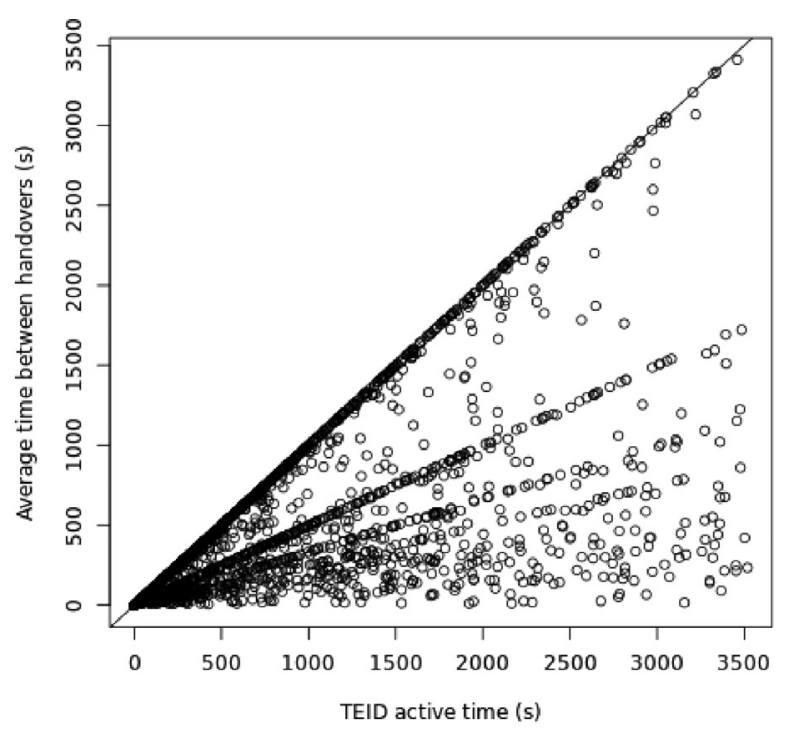

(b) Peak hour

Fig. 3. Duration of tunnel identifiers vs. average of the time between handovers for the low and peak hours.

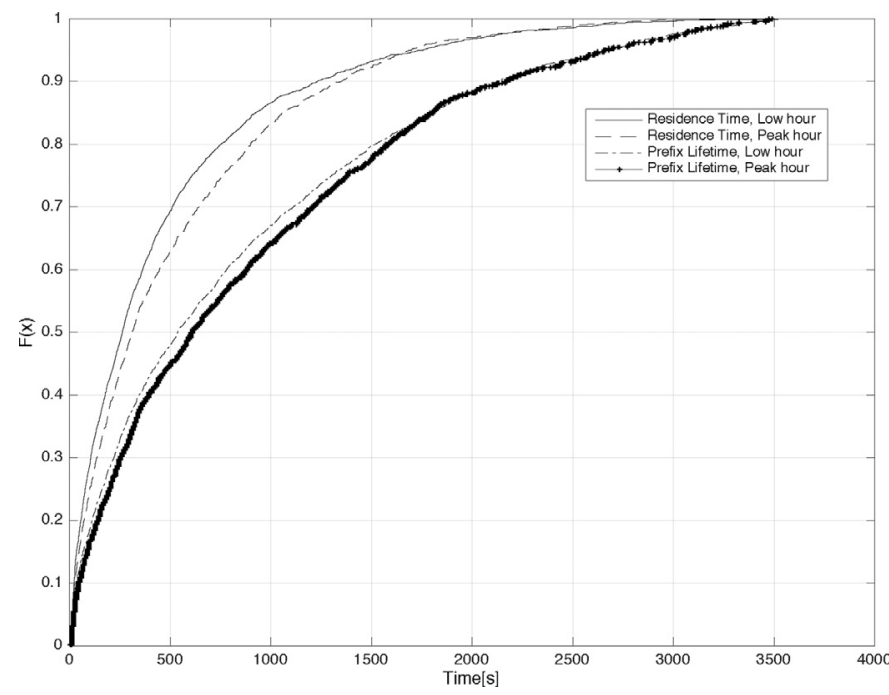

Fig. 4. Comparison of the residence time and prefix lifetime for the peak and low hours (CDF).

the mobility requirements, we claim that mobility can be managed more efficiently.

\subsection{PMIPv6}

PMIPv6 is a network-based protocol that provides localized mobility management [36]. Its operation is conceptually identical to the one currently used in cellular networks, GTP. PMIPv6 releases the mobile terminal from its involvement in the mobility management of traditional IP mobility protocols, to place it at the network entities: the Local Mobility Anchor (LMA) and the Mobile Access Gateway (MAG), as illustrated in Fig. 5a. The LMA is placed at the operator's core network and manages the routing for all the mobile nodes within the mobility domain. Traffic for each mobile node is tunneled between the LMA and the corresponding MAG, through an IP in IP tunnel. The MAG performs all the mobility management signaling with the LMA on behalf of the mobile node by the exchange of Proxy Binding Update (PBU) and Proxy Binding Acknowledgment (PBA) messages. The MAG is the access router of the mobile node, that is, it acts as the first IP hop in the mobility domain. In case of handover, the target MAG signals the LMA upon the attachment of the mobile node, establishing a new tunnel and eliminating the tunnel to the former MAG. The main problem of such a hierarchical architecture is the scalability, since all traffic must traverse the GGSN (LMA) which constitutes a bottleneck and single point of failure. In addition all traffic is treated the same and is provided with mobility management (is tunneled from LMA to $M A G)$.

\subsection{Network-based DMM}

DMM proposes a flatter architecture, placing the mobility management entities closer to the mobile node. DMM solutions are still being discussed at the IETF. ${ }^{5}$ For this work we consider our proposal in [37] as the basis for DMM operation, which is illustrated in Fig. 5b. The roles of LMA and MAG are absorbed by a new entity, the Mobility Anchor and Access Router (MAAR). The MAAR acts as the Access Router for the mobile node, handles its mobility and the routing. Although there are different approaches, part of the functionality of the LMA is covered by a node that stores the prefixes assigned to every mobile node, mainly as a Central Mobility Database (CMD in Fig. 5b). In the case of handover, the target MAAR establishes a tunnel to the former MAAR to forward the traffic for the mobile node's previous prefix, while traffic not requiring mobility is forwarded directly to the Internet. Complexity increases as the mobile node performs more handovers, but in the general case DMM results in a more efficient and scalable approach for mobility management. One of the key aspects of DMM is that the flows originated by the mobile terminal are not tunneled, but only the flows performing handovers require the overhead of tunneling through the network. For networks with low mobility characteristics, this is a key difference, since most of flows will not require differentiated handling from standard IP routing, reducing the overhead in the network.

\footnotetext{
${ }^{5}$ http://datatracker.ietf.org/wg/dmm/documents/
} 


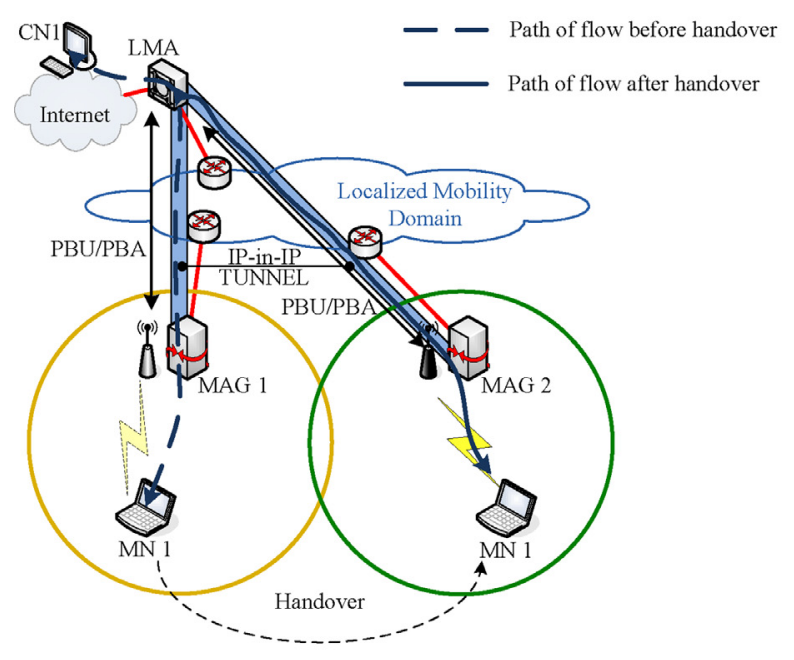

(a) PMIPv6 operation

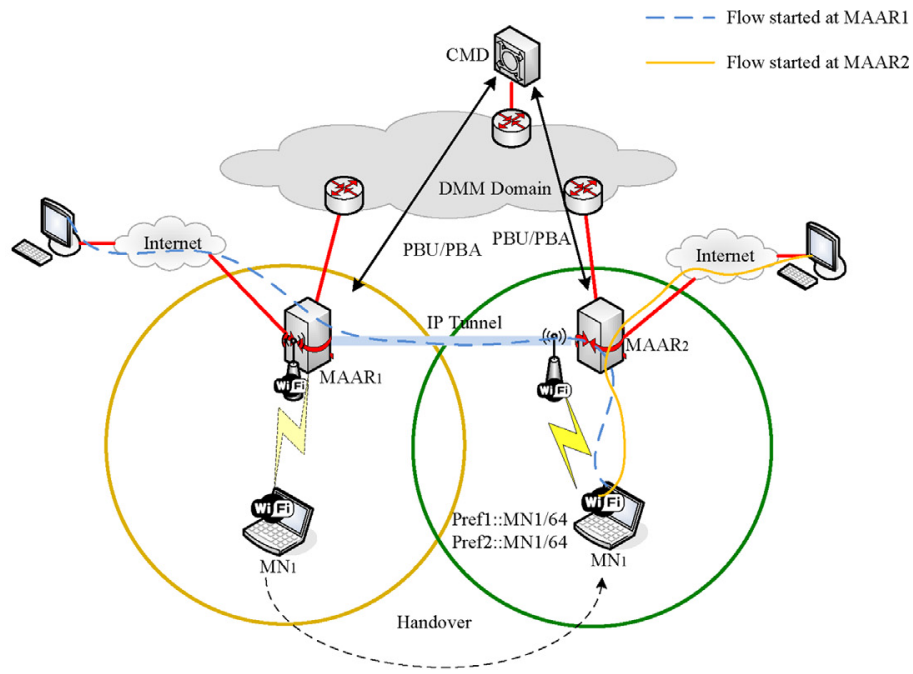

(b) Network-based DMM operation

Fig. 5. Operation of PMIPv6 and network-based DMM protocols.

\subsection{DMM deployment analysis}

Based on our previous analysis published in [38], we compare the packet delivery gains attained by the use of DMM principles. In a DMM architecture, the number of active prefixes (for relating to our scenario, active tunnels) is directly related to the number of handovers performed by the mobile node. In [38], it is proven that the gains of DMM over PMIPv6 are given by Eq. (1), where $\bar{N}_{P R}$ is the average number of active prefixes at a handover and $C_{M A A R-M A A R}$ and $C_{L M A-M A G}$ are expressed in terms of delay between MAARs and between LMA-MAG respectively

$\frac{C_{D M M}}{C_{P M I P}}=\left(\bar{N}_{P R}-1\right) \frac{C_{M A A R-M A A R}}{C_{L M A-M A G}}$

The data on which we base our analysis comes from a $\mathrm{Gn}$ interface and corresponds to traffic between a GGSN and a single SGSN that handles several routing areas. We propose the scenario in Fig. 6 to compare with PMIPv6 and DMM approaches. We can see that PMIPv6 is conceptually similar to GTP, and the packet delivery cost comparison between DMM and PMIPv6 can be similarly extended to GTP. We propose as a reasonable scenario to have the LMA co-located with the GGSN and the MAG with the SGSN. For the DMM architecture, we propose to place a MAAR in every RA (i.e. RNC). Therefore, to follow the analysis given by Eq. (1) the number of active prefixes applying DMM to our scenario is given by the number of tunnels performing a RA change. As the analysis in [38] involves the average number of prefixes active per user and we have the aggregated information of active tunnels in the network, for comparison with PMIPv6, we need to take into account all the active tunnels in the network (which is equal to 1 active prefix per user in the analysis provided in [38]). We have tracked the RA changes per tunnel, as reported in Section 3.4, to find out that the average number of handovers per TEID in the network is 1.8 and the total number of tunnels performing at least one handover is 1464 for the low hour and 2413 for the peak hour (roughly $2 \%$ of the tunnels in the network for both cases). With this information and the number of packets delivered per tunnel, we can say that the packet delivery cost ratio is given by Eq. (2), where $\lambda_{\text {mov }}$ is the number of data packets sent in the tunnels that experienced handover, $\lambda_{\text {tot }}$ is the total number of data packets, $N_{\text {mov }}$ is the number of tunnels that experience handover and $N_{\text {tot }}$ is the total number of tunnels. This is equivalent to the ratio between the traffic that experiences a handover (and should be provided mo-
Table 2

Value of the parameters in $\mathrm{Eq} .(2)$

\begin{tabular}{lrr}
\hline & Low hour & Peak hour \\
\hline$\lambda_{\text {mov }}$ & 41467 & 49927 \\
$\lambda_{\text {tot }}$ & 18978264 & 28582691 \\
$N_{\text {mov }}$ & 1464 & 2413 \\
$N_{\text {tot }}$ & 60932 & 116529 \\
\hline
\end{tabular}

bility) and the total amount of traffic being served in the network. Note that, for $\lambda_{\text {mov }}$ we are considering a worst case scenario, as the amount of data packets that would be tunneled by DMM would be the packets that are sent after the handover, but not in the routing area in which the flow is originated

$$
\frac{C_{D M M}}{C_{P M I P}}=\frac{\lambda_{\text {mov }} / N_{\text {mov }}}{\lambda_{\text {tot }} / N_{\text {tot }}} \frac{D_{\text {MAAR-MAAR }}}{D_{\text {IMA-MAG }}}
$$

The values for the two datasets analyzed to be substituted in Eq. (2) are shown in Table 2. Applying these results to Eq. (2) for the peak hour trace we obtain

$\frac{C_{D M M}}{C_{P M I P}}=0.08 \frac{D_{M A A R-M A A R}}{D_{\text {LMA-MAG }}}$

Computing the delay between MAARs that makes equal the packet delivery cost of applying PMIPv6 and DMM, we have for the low and peak hours respectively

$$
\begin{aligned}
& D_{M A A R-M A A R}^{\text {peak_hour }}=11.85 D_{L M A-M A G} \\
& D_{M A A R-M A A R}^{\text {low_hour }}=10.99 D_{L M A-M A G}
\end{aligned}
$$

This result can be explained given the low mobility in the network. For instance, in the peak hour case, the number of tunnels that are provided mobility is so low compared to the total amount of traffic in the network that the distance between MAARs (in terms of delay) can be up to 11.85 times the distance between LMA and MAG for the packet delivery cost of DMM and PMIPv6 to be equal. These results show this network exhibits the perfect traffic patterns for the deployment of DMM mobility, i.e., low mobility of users with short life sessions.

It is important to analyze the meaning of these results at the light of the different kind of users present in the network. The above results are obtained for the overall traffic, which is characterized by a low amount of flows actually moving. The results 


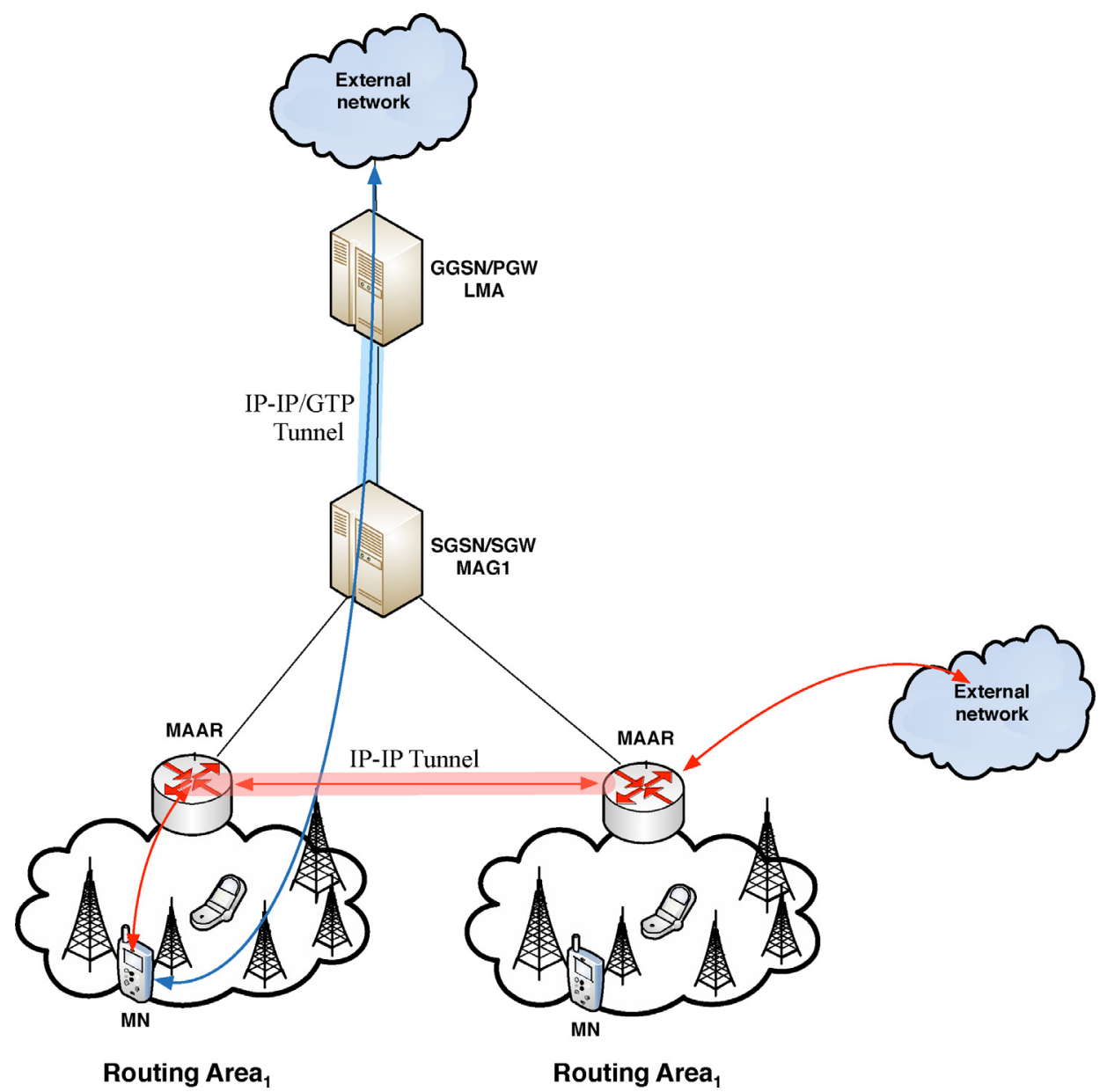

Fig. 6. Cellular network architecture with PMIPv6 and DMM mobility entities co-located.

suggest that the operator can deploy a DMM solution by replacing its LMA by a network of DMM MAARs separated by e.g., 11 times the current latency between the LMA and MAG, while keeping the packet delivery cost equal to the current one. This is mainly due to a reduced cost for the transport of traffic generated by non-mobile users. The savings come from the fastest off-loading of traffic to the Internet without crossing the operator core and a reduced overhead. ${ }^{6}$ The downside of this approach is that mobile packets will incur in a higher cost, which is compensated by the optimization of the non-mobile users. In order to quantify this side effect, we have extended the above analysis considering only the mobile users data.

Previous work in [38] models the residence time and the prefix lifetime as Gamma and Exponential functions respectively. Based on the approximate probability functions of these metrics we are able to compute the average number of prefixes surviving handovers for the traffic of users actually moving.

Figs. $7 \mathrm{a}$ and $7 \mathrm{~b}$ show the fitting of the measured data and the probability distribution functions, while Table 3 presents the parameters resulting from the function fitting process.

Based on these parameters, we can apply the model developed in [38] to compute the average number of prefixes that a mobile user must maintain alive while performing handover (for non

\footnotetext{
${ }^{6}$ It is worth noting that with PMIP or GTP solutions, all traffic is encapsulated in a tunnel and traverses the network between the LMA and MN. On the contrary, for the DMM solution, traffic of users not moving does not suffer any encapsulation and is directly forwarded to the Internet without traversing the operator core.
}

Table 3

Probability density function parameters.

\begin{tabular}{lll}
\hline & PDF \\
\hline Gamma & $\frac{\gamma \mu_{S N}^{\gamma}}{\Gamma(\gamma)} t^{\gamma-1} e^{-\gamma \mu_{S N} t}$ & \\
Exponential & $\lambda_{P R} e^{-x \lambda_{P R}}$ & \\
& Parameters & Peak hour \\
\cline { 2 - 3 } & Low hour & $\gamma=0.7483$ \\
\hline Gamma & $\gamma=0.8033$ & $1 / \mu_{S N}=464.72$ \\
Exponential & $1 / \mu_{S N}=522.1$ & $\lambda_{P R}=838.058$ \\
\hline
\end{tabular}

static users), as shown in Eq. (6).

$N_{P R}=\frac{\left(\lambda_{P R}+\gamma \mu_{S N}\right)^{\gamma}}{\left(\lambda_{P R}+\gamma \mu_{S N}\right)^{\gamma}-\left(\gamma \mu_{S N}\right)^{\gamma}}$

Using the parameters shown in Table 3 we obtain the following values of $N_{P R}$ for the peak and low hours:

$N_{P R}^{\text {lowhour }}=2.79$,

$N_{P R}^{\text {peakhour }}=2.9$.

Following the same procedure as with Eqs. (4) and (5), we can estimate the distance between MAARs in order to obtain the same packet delivery cost as with current deployment:

$D_{M A \bar{A}-\text { hour }}^{\text {lowAR }}=0.55 D_{L M A-M A G}$ 


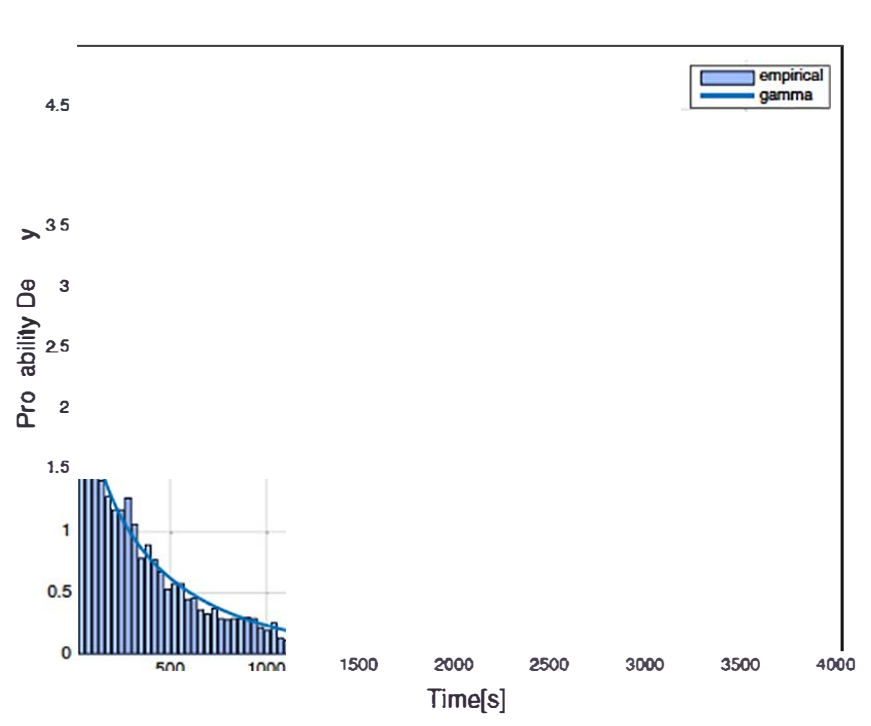

(a) Residence Time.

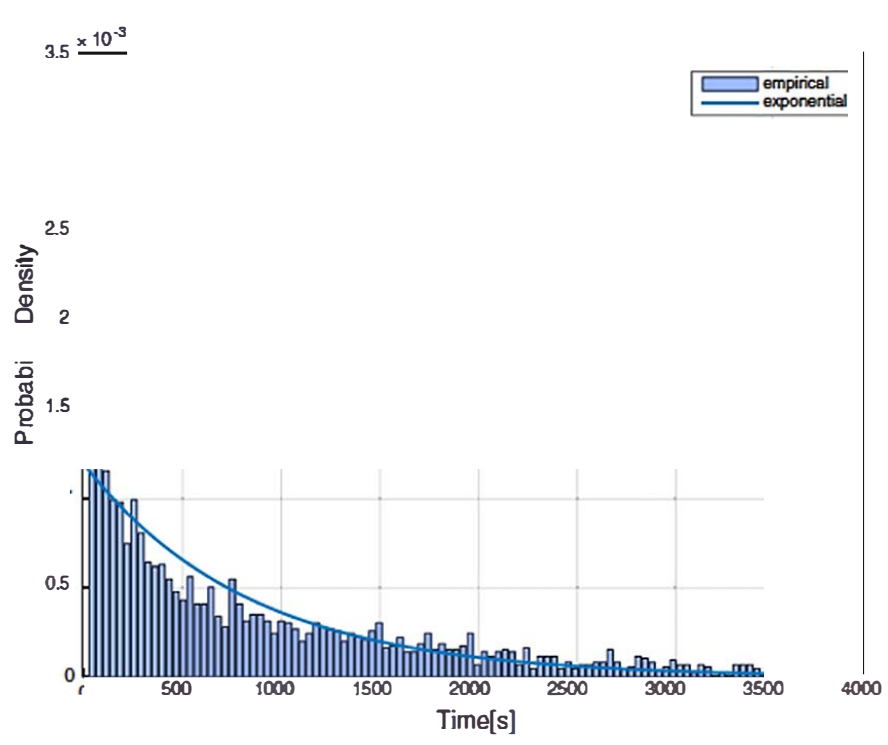

(b) IP prefix lifetime.

Fig. 7. Gamma and exponential distribution approximation for the residence time and IP prefix lifetime (peak hour).

$D_{\text {MAAR-MAAR }}^{\text {peak hour }}=0.53 D_{\text {LMA-MAC }}$

Clearly, the level of flattening that can be applied to the network considering only the mobile traffic is much lower than the one achievable considering the complete set of traffic sources. In order to assess the impact on the packet delivery cost of flattening the network at the levels shown in Eqs. (4) and (5), we can simply substitute the ratio $\frac{D_{\text {MAR }} \text {-MAAR }}{D_{\text {LMA }} \text { baG }}$ by the value computed for the complete data set, obtaining the following value (only computed for the peak hour):

$\frac{C_{D M M}}{C_{P M I P}}=23.5$.

This result shows that the high flattening ratio computed in Eqs. (4) and (5) produces an increased cost on the delivery of packets generated by mobile users. If we consider the reverse situation, we could dimension the network in order to optimize the mobile traffic delivery cost. In this case, we need to consider the value of the ratio $\frac{D_{\text {MARR }} \text {-MAAR }}{D_{\text {LMA }} \text {-MAG }}$ that makes the ratio of costs equal to one and substitute this value in Eq. (3), yielding the following result:

$C_{\text {DMM }}=0.0416$.

This result is quite interesting and shows how a MAAR deployment based on the traffic pattern of only the mobile users $\left({ }^{D_{\text {MAAR-MAAR }}}=0.5\right)$ will result on a global significant reduction of the packet delivery cost for the complete network.

To conclude this section we analyze the signaling load required to support DMM compared with PMIP. As explained before, DMM approaches typically require a higher signaling load compared with traditional approaches such as PMIP or GTP, but this overhead is only required for the traffic experimenting a handover. For traffic not moving, it is not required any kind of signaling since flows anchored in the MAARs never leave it. Following the analysis in [38], the signaling cost ratio between PMIP and DMM can be computed as follows:

$$
\frac{C_{s i g}^{D M M}}{C_{s i g}^{P M I P}}=1+\begin{gathered}
1+N_{p_{R}} \\
2+\left\lfloor 1 / \mu_{S N} T_{B C E}\right\rfloor
\end{gathered}
$$

where $T_{B C E}$ corresponds to the Binding Cache Entry lifetime and is usually configured to $300 \mathrm{~s}$.

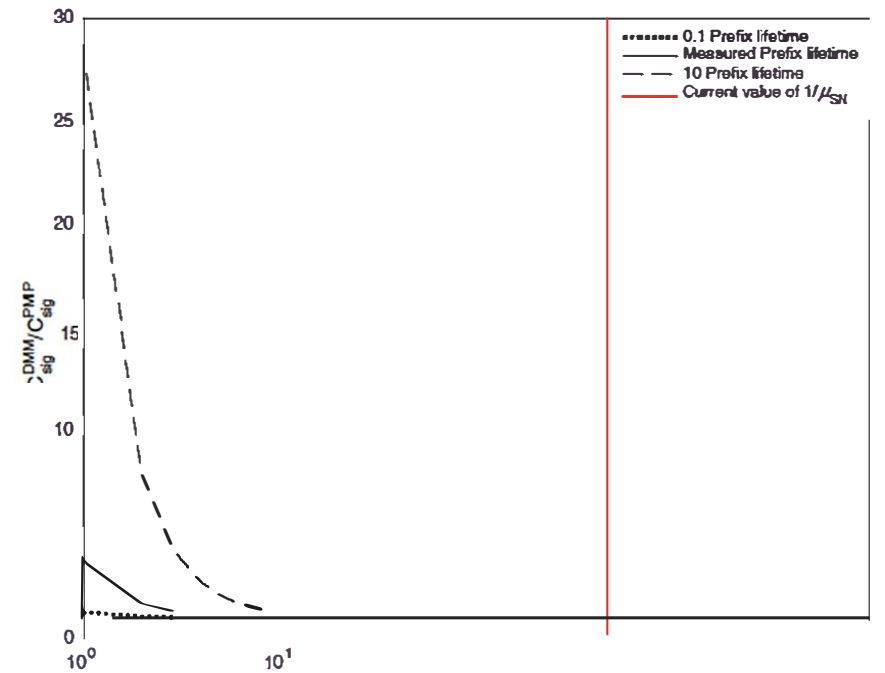

Fig. 8. Signaling cost comparison for DMM and PMIP.

Fig. 8 shows the ratio between the signaling costs for DMM and PMIP for different values of the residence time $\left(1 / \mu_{S N}\right)$ and prefix lifetime $\left(\lambda_{P R}\right)$. Note that this figure does not include the signaling load for the static users in case of PMIP, i.e., the analysis is performed with the derived distributions presented in Table 3. Results indicate the current traffic profile will yield a similar signaling cost for PMIP and DMM. As mobility increases (reduction of $1 / \mu_{S N}$ ) the cost of the DMM solution skyrockets, since for each movement several prefixes/previous DMM MAARs must be updated with the new location of the user. This effect is exacerbated when the average session lifetime increases. The longer a session is kept open in a user terminal, the worst for the DMM signaling, since the number of handover the application flow will survive will increase. Fortunately, mobile applications are not following this trend, using 
several parallel short-lived flows to download the content into the terminal. $^{7}$

The analysis presented on this section, based on real traces from operator's network, indicates how the anchor points (MAARs) of a distributed mobility management solution should be deployed considering its users' traffic characteristics and how the foreseen increment of the prefix lifetime and reduction of the residence time affect the cost of signaling of DMM compared with PMIP. We claim the use of this technology is key for the development of $5 G$ network architectures, characterized by extreme consumption of resources in the network. The mobility in the network for the two loads studied is around $2 \%$, despite the amount of traffic served in the system. This favors the deployment of a flatter architecture like DMM as the traffic that requires mobility is a small portion of the total. In addition, TEIDs, in average do not experience a high number of RA changes, so the number of tunnels that should be maintained active in a DMM solution would be much lower.

\section{Conclusion}

In this work, we collect the information observed in the Gn interface between the core network nodes and extract handoverrelated information for further analysis from a real operator's network. The extraction steps, which can be applied to all mobile networks using 3GPP standard, are provided in detail. We match the location information present in the control plane, which is missing in the user plane packets to evaluate mobility in the network thanks to big data platform and analyze the mobility requirements. Our mobility analysis reveals that a DMM-based mobility solution, flattening the current deployment by a factor of almost 11, can be applied. The new mobility management solution will provide increased scalability to the operator network, without any additional overhead. The limits on the profitability of this solution will depend on the specific characteristics of the operator's network and the mobile data traffic mobility. As future work we plan to extend the period of time analysed, searching for time patterns and providing guidelines for deployment of mobility solutions.

\section{Acknowledgment}

The research leading to these results has received funding from the EU Seventh Framework Programme (FP7/2007-2013) under grant agreement 318115 (Connectivity management for eneRgy Optimised Wireless Dense networks, CROWD). The work of Antonio de la Oliva has also been funded by the EU H2020 5G-Crosshaul Project (grant no. 671598).

\section{References}

[1] A. Imran, A. Zoha, Challenges in 5G: how to empower SON with big data for enabling 5G, Netw., IEEE 28 (6) (2014) 27-33, doi:10.1109/MNET.2014.6963801.

[2] F. Ricciato, E. Hasenleithner, P. Romirer-Maierhofer, Traffic analysis at short time-scales: an empirical case study from a 3G cellular network, Netw. Serv. Manage. IEEE Trans. 5 (1) (2008) 11-21, doi:10.1109/TNSM.2008.080102.

[3] J. Liu, F. Liu, N. Ansari, Monitoring and analyzing big traffic data of a largescale cellular network with Hadoop, Netw. IEEE 28 (4) (2014) 32-39, doi:10. 1109/MNET.2014.6863129.

[4] J. Zuniga, C. Bernardos, A. De La Oliva, T. Melia, R. Costa, A. Reznik, Distributed mobility management: a standards landscape, Commun. Mag. IEEE 51 (3) (2013) 80-87, doi:10.1109/MCOM.2013.6476870.

[5] R. Bolla, M. Repetto, A comprehensive tutorial for mobility management in data networks, Commun. Surv. Tut. IEEE 16 (2) (2014) 812-833, doi:10.1109/ SURV.2013.071913.00140.

[6] J. Arkko, C. Perkins, D. Johnson, Mobility support in IPv6, 2011, (IETF RFC 6275).

\footnotetext{
${ }^{7}$ One example of this trend is the Dynamic Adaptive Streaming over HTTP (DASH), which segments videos in different chunks that are downloaded by
}

[7] V. Devarapalli, K. Chowdhury, B. Patil, Proxy mobile IPv6, 2008, (IETF RFC 5213).

[8] H. Soliman, C. Castelluccia, K. ElMalki, L. Bellier, Hierarchical mobile IPv6 (HMIPv6) mobility management, 2008, (IETF RFC 5380).

9] 3GPP TS 29.060: General Packet Radio Service (GPRS); GPRS Tunnelling Protocol (GTP) across the Gn and Gp interface, 2013, (http://www.3gpp.org/ DynaReport/29060.htm).

[10] H. Chan, (Ed.), Requirements for distributed mobility management, 2014. (IETF RFC 7333).

[11] K.-H. Lee, H.-W. Lee, W. Ryu, Y.-H. Han, A scalable network-based mobility management framework in heterogeneous ip-based networks, Telecommun. Syst. 52 (4) (2013) 1989-2002, doi:10.1007/s11235-011-9479-3.

[12] IETF charter for working group DMM, 2015, (https://datatracker.ietf.org/wg/ dmm/charter/). [[Online] Available; accessed 25-November-2015].

[13] F. Giust, L. Cominardi, C. Bernardos, Distributed mobility management for future 5G networks: overview and analysis of existing approaches, Commun. Mag. IEEE 53 (1) (2015) 142-149, doi:10.1109/MCOM.2015.7010527.

[14] E. Halepovic, C. Williamson, Characterizing and modeling user mobility in a cellular data network, in: Proceedings of the 2nd ACM international workshop on Performance evaluation of wireless ad hoc, sensor, and ubiquitous networks, ACM, 2005, pp. 71-78.

[15] U. Paul, A.P. Subramanian, M.M. Buddhikot, S.R. Das, Understanding traffic dynamics in cellular data networks, in: INFOCOM, 2011 Proceedings IEEE, IEEE, 2011, pp. 882-890.

[16] F. Calabrese, F.C. Pereira, G. Di Lorenzo, L. Liu, C. Ratti, The geography of taste: analyzing cell-phone mobility and social events, in: Pervasive computing, Springer, 2010, pp. 22-37.

[17] M.Z. Shafiq, L. Ji, A.X. Liu, J. Wang, Characterizing and modeling internet traffic dynamics of cellular devices, in: Proceedings of the ACM SIGMETRICS Joint International Conference on Measurement and Modeling of Computer Systems, ACM, 2011, pp. 305-316.

[18] Q. Xu, Y. Liao, S. Miskovic, Z.M. Mao, M. Baldi, A. Nucci, T. Andrews, Automatic generation of mobile app signatures from traffic observations, in: Computer Communications (INFOCOM), 2015 IEEE Conference on, IEEE, 2015 pp. 1481-1489.

[19] R.W. Douglass, D.A. Meyer, M. Ram, D. Rideout, D. Song, High resolution population estimates from telecommunications data, EPJ Data Sci. 4 (1) (2015) 1-13.

[20] S. Hoteit, S. Secci, S. Sobolevsky, C. Ratti, G. Pujolle, Estimating human trajectories and hotspots through mobile phone data, Comput. Netw. 64 (2014) 296-307.

[21] D. Naboulsi, M. Fiore, S. Ribot, R. Stanica, Large-scale mobile traffic analysis: a survey, IEEE Commun. Surv. Tut. 18 (2015) 124-161.

[22] Apache Hadoop project, 2015, (http://hadoop.apache.org/), [Online; accessed 02-April-2015].

[23] O. Celebi, E. Zeydan, O. Kurt, O. Dedeoglu, O. Iieri, B. AykutSungur, A. Akan, S. Ergut, On use of big data for enhancing network coverage analysis, in: Telecommunications (ICT), 2013 20th International Conference on, 2013, pp. 15, doi:10.1109/ICTEL.2013.6632155.

[24] A. Karatepe, E. Zeydan, Anomaly detection in cellular network data using big data analytics, in: European Wireless 2014; 20th European Wireless Conference; Proceedings of, 2014, pp. 1-5.

[25] E. Batug, M. Bennis, E. Zeydan, M. Kader, A. Karatepe, A. Salih Er, M. Debbah, Big data meets telcos: a proactive caching perspective, Journal of Commun. Netw. 17 (2015) 549-557.Special Issue on Big Data Networking-Challenges and Applications

[26] M. Abdel Kader, E. Batug, M. Bennis, E. Zeydan, A. Karatepe, A. Salih Er, M. Debbah, Leveraging big data analytics for cache-enabled wireless networks, in: IEEE Global Communications Conference (GLOBECOM) Workshop; Proceedings of, 2015

[27] J. Magnusson, T. Kvernvik, Subscriber classification within telecom networks utilizing big data technologies and machine learning, in: Proceedings of the $1 \mathrm{st}$ International Workshop on Big Data, Streams and Heterogeneous Source Mining: Algorithms, Systems, Programming Models and Applications, in: BigMine '12, ACM, New York, NY, USA, 2012, pp. 77-84, doi:10.1145/2351316.2351327.

[28] A. Sultan, F. Benbadis, V. Gauthier, H. Afifi, Mobile data network analysis platform, in: Proceedings of the 6th International Workshop on Hot Topics in Planet-Scale Measurement, in: HotPlanet '15, ACM, New York, NY, USA, 2015, pp. 13-18, doi:10.1145/2798087.2798093.

[29] F. Ricciato, R. Pilz, E. Hasenleithner, Measurement-Based Optimization of a 3C Core Network: a Case Study, in: Y. Koucheryavy, J. Harju, V. Iversen (Eds.), Next Generation Teletraffic and Wired/Wireless Advanced Networking, Lecture Notes in Computer Science, vol. 4003, Springer, Berlin Heidelberg, 2006, pp. 70-82, doi:10.1007/11759355 9.

[30] Cloudera, 2015, (http://www.cloudera.com/content/cloudera/en/ documentation.html), [Online; accessed 02-April-2015].

[31] X. He, P.P. Lee, L. Pan, C. He, J.C. Lui, A panoramic view of $3 g$ data/control-plane traffic: mobile device perspective, in: NETWORKING 2012, Springer, 2012, pp. 318-330.

[32] W. Hahn, 3GPP Evolved Packet Core support for distributed mobility anchors: Control enhancements for GW relocation, in: ITS Telecommunications (ITST), 2011 11th International Conference on, 2011, pp. 264-267, doi:10.1109/ITST. 2011.6060065.

[33] 3GPP TS 23.401, General packet radio service (gprs) enhancements for evolved universal terrestrial radio access network (e-utran) access, Rel. 12, (http:// www.3gpp.org/DynaReport/23401.htm). 
[34] Ruckus, Interworking wi-fi and mobile networks, 2013, (http://c541678.r78.cf2. rackcdn.com/wp/wp-interworking-wi-fi-and-mobile-networks.pdf), White Paper, [Online; accessed 01-March-2016].

[35] , Cisco / Starent Networks, LTE: simplifying the migration to 4G networks, 2010. http://www.cisco.com/c/dam/en/us/solutions/service-provider/ mobile-internet/whitepaper_c11-577763_v1.pdf.

[36] S. Gundavelli, K. Leung, V. Devarapalli, K. Chowdhury, B. Patil, Proxy Mobile IPv6, 2008, (RFC 5213 (Proposed Standard)).
[37] C. Bernardos, A. de la Oliva, F. Giust, A PMIPv6-based solution for distributed mobility management, 2014, (Internet-Draft (work in progress), draftbernardos-dmm-pmip-03).

[38] F. Giust, C. Bernardos, A. De La Oliva, Analytic evaluation and experimental validation of a network-based IPv6 distributed mobility management solution, Mobile Comput. IEEE Trans. 13 (11) (2014) 2484-2497, doi:10.1109/TMC.2014 2307304. 